\title{
Electrochemical Pseudo-Titration of Water-Soluble Antioxidants
}

\section{-- Supporting Information -}

Philippe Tacchini, ${ }^{\mathrm{a}}$ Andreas Lesch, ${ }^{\mathrm{b}}$ Alice Neequaye, ${ }^{\mathrm{a}}$ Grégoire Lagger, ${ }^{\mathrm{a}, \mathrm{c}}$ Jifeng Liu, ${ }^{\mathrm{d}}$ Fernando Cortés-Salazar, Hubert H Girault, ${ }^{\mathrm{b}, *}$

a EDEL Therapeutics SA, PSE-B, EPFL, CH-1015 Lausanne, Switzerland

b Laboratoire d'Electrochimie Physique et Analytique, Station 6, Ecole Polytechnique Fédérale de Lausanne, CH-1015 Lausanne, Switzerland

c Service d'Enseignement Thérapeutique pour Maladies Chronique, Faculté de Médecine, Université de Genève, CH-1205 Genève, Switzerland

d Department of Chemistry, Liaocheng University, No.1 Hunan Road, Liaocheng, Shandong Province, 252059, China

* e-mail: hubert.girault@epfl.ch

Tel: +41216933145; Fax: +41216933667.

\section{Table of contents}

SI-1 Cascade oxidation of multi-component systems during linear sweep voltammetry

SI-2 Optimisation of $\lambda$ and $E_{\text {threshold }}$

SI-3 Principle of pseudo-titration for a multi-component system

SI-4 Triplicate measurement of AOP of blood 


\section{SI-1 Cascade oxidation of multi-component systems during linear sweep voltammetry}

Natural samples usually consist of a large variety of redox active species. Most likely, the electrochemical oxidation of these compounds take place at different anodic potentials and the speed of the global reaction will depend on different heterogeneous reaction rates. Therefore, a linear sweep will show the sum of the individual current-potential curves, which results in an increasing wave-shaped current (curve 1 in Figure SI-1). This curve was constructed by the addition of three linear sweep voltammograms (LSVs) of three redox active species A, B and C whose formal redox potentials and standard reaction rates differ significantly.

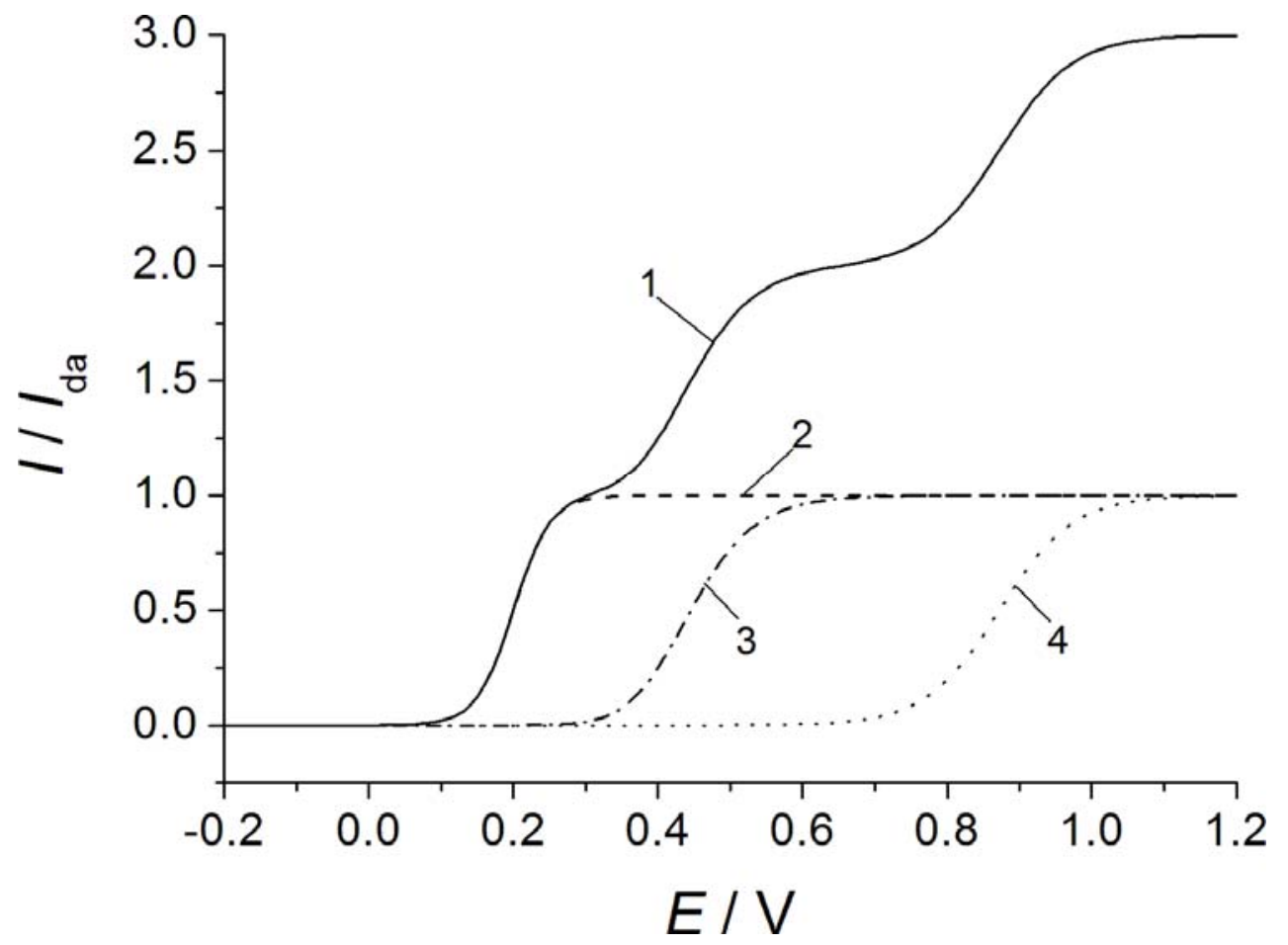

Fig. SI-1.Simulated linear sweep voltammograms demonstrating the cascade oxidation of a multicomponent system (curve 1). This plot was built by adding the LSVs of three compounds A, B and C: $k_{\mathrm{A}}^{0}=10 \mathrm{~cm} \cdot \mathrm{s}^{-1}, E_{\mathrm{A}}{ }_{\mathrm{A}}=0.2 \mathrm{~V}(2) ; k_{\mathrm{B}}^{0}=0.01 \mathrm{~cm} \cdot \mathrm{s}^{-1}, E_{\mathrm{B}}{ }_{\mathrm{B}}=0.45 \mathrm{~V}(3) ; k_{\mathrm{C}}^{0}=0.0001 \mathrm{~cm} \cdot \mathrm{s}^{-1}, E^{\prime}{ }_{\mathrm{C}}=0.88 \mathrm{~V}$ (4). $D=10 \cdot 10^{-5} \mathrm{~cm}^{2} \mathrm{~s}^{-1}, \delta=5 \cdot 10^{-4} \mathrm{~cm}, \alpha=0.5$. 


\section{SI-2 Optimisation of $\lambda$ and $E_{\text {threshold }}$}

In order to optimise the modulated current with respect to the pseudo-titration voltammetry (PTV) curves, the threshold potential is usually selected to be slightly higher than the formal redox potential of the investigated redox species. In this theoretical example using the same parameters as in Figure 2 of the main manuscript, $E_{\text {threshold }}$ was set to $0.8 \mathrm{~V}\left(E^{\prime}=0.6 \mathrm{~V}\right)$. Figure SI-2a shows the normalised currentpotential curves and the Fermi-Dirac functions with $\lambda=1,0.5$ and 2.5. The PTVs are shown in Figure SI-2b. Due to the shift of $E_{\text {threshold }}$ the modulated currents are significantly higher. Also reactions with slow kinetics (curve 5) are detected. The peaks become sharper with decreasing $\lambda$, but contrarily the influence of slow kinetics increases. These results demonstrate that a compromise between $E_{\text {threshold }}$ and $\lambda$ has to be determined.

a)
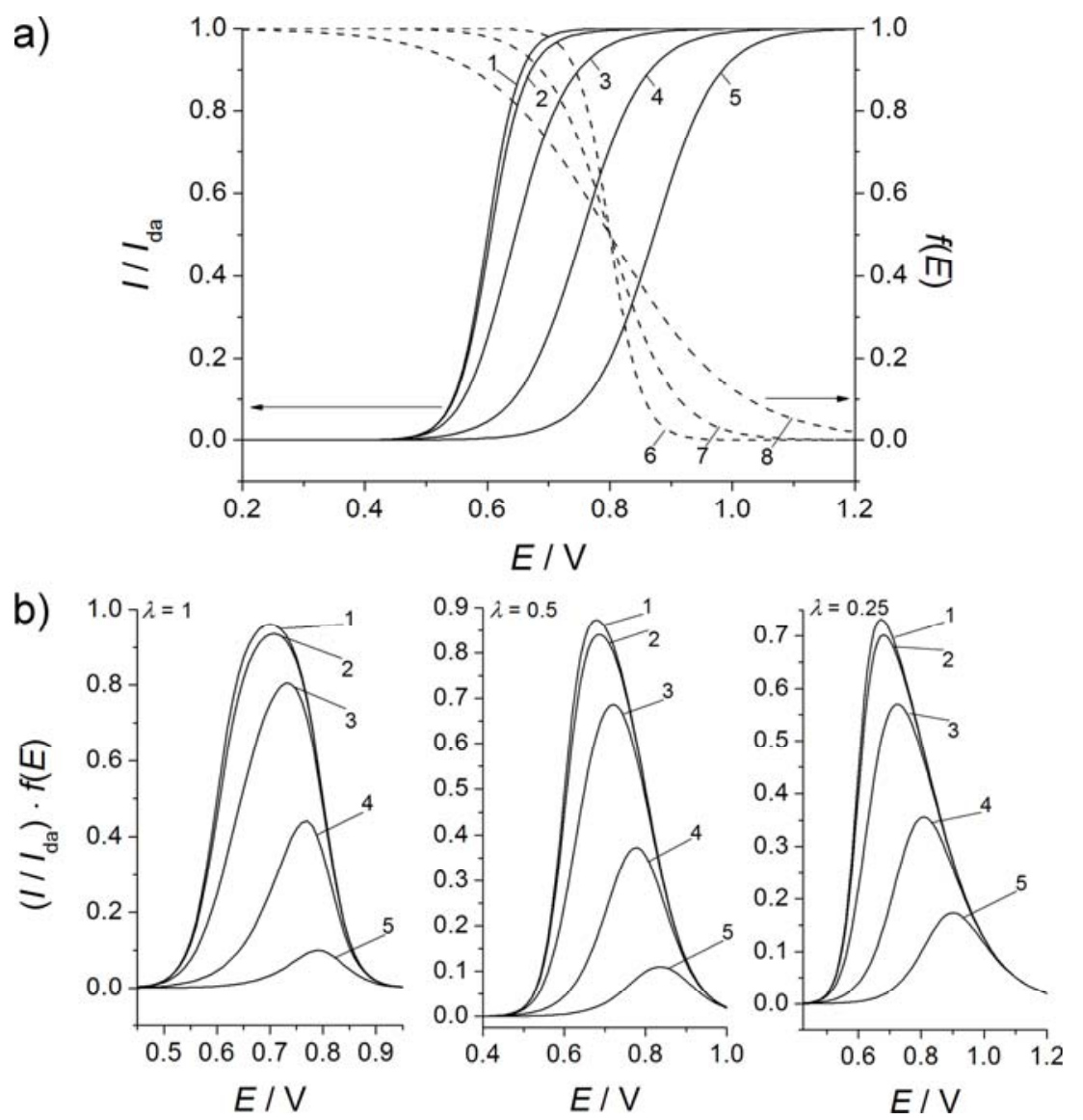

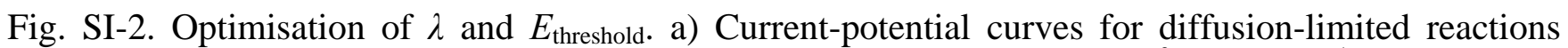
(solid lines, Equation (1)) using the same different standard reactions rates: $k^{0}=10 \mathrm{~cm} \cdot \mathrm{s}^{-1}$ (curve 1), 0.1 $\mathrm{cm} \cdot \mathrm{s}^{-1}(2), 0.01 \mathrm{~cm} \cdot \mathrm{s}^{-1}$ (3), $0.001 \mathrm{~cm} \cdot \mathrm{s}^{-1}$ (4) and $0.0001 \mathrm{~cm} \cdot \mathrm{s}^{-1}$ (5). $D=10 \cdot 10^{-5} \mathrm{~cm}^{2} \cdot \mathrm{s}^{-1}, \delta=5 \cdot 10^{-4} \mathrm{~cm}, \alpha=$ $0.5, E^{\prime}=0.6 \mathrm{~V}$. Fermi-Dirac plots of Equation (3) (dashed lines) using different $\lambda$ and $E_{\text {threshold }}=0.8 \mathrm{~V}$. $\lambda=1$ (curve 6), 0.5 (7) and 0.25 (8). b) Pseudo-titrated curves. 


\section{SI-3 Principle of pseudo-titration voltammetry for a multi-component system}

The PTV of the multi-component system introduced in SI-1 clearly demonstrates how reactions with high $E$ ' and slow kinetics are completely suppressed (Figure SI-3) whereas the fast reactions (i.e. the AOs in a biological system) appear as a peak. However, the peak height and shape can be influenced by $E_{\text {threshold. }} E^{\prime}$ of the desired reaction is $0.2 \mathrm{~V}$. A $E_{\text {threshold }}$ of $0.4 \mathrm{~V}$ gave a larger peak than $0.2 \mathrm{~V}$. However, the influence of the second reaction has to be taken into consideration.

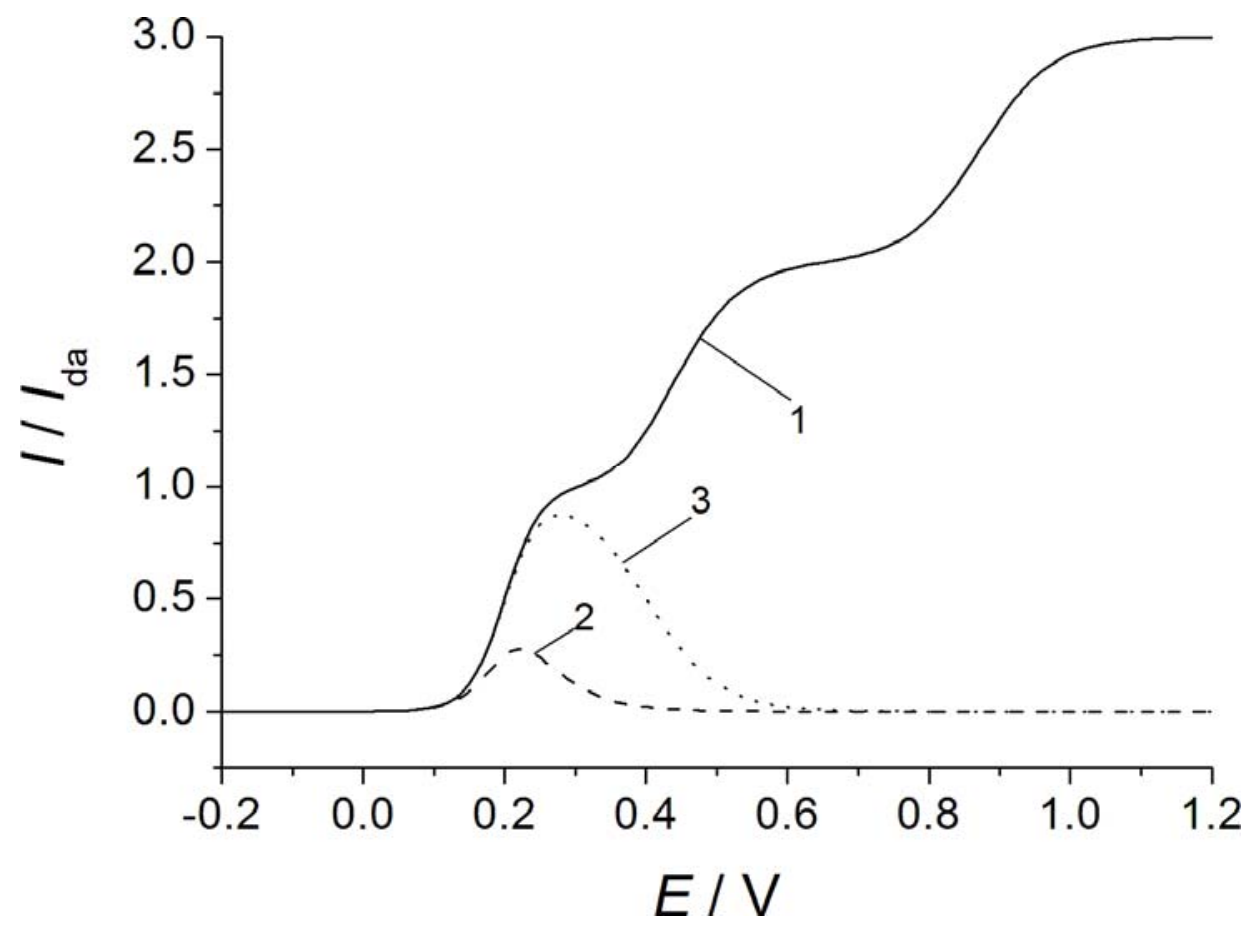

Fig. SI-3. Simulated linear sweep voltammogram of a cascade oxidation of a multi-component system (curve 1, same curve as in Fig. SI-1). PTVs with $\lambda=0.5$ and $E_{\text {threshold }}=0.2 \mathrm{~V}$ (2) and $0.4 \mathrm{~V}$ (3). 


\section{SI-4 Triplicate measurement of AOP of blood}

LSVs of blood from a female test person were recorded in a triplicate measurement. The original LSVs are shown in Figure SI-4a and the PTVs in Figure SI-4b. The AOP values were calculated and are listed in Table SI-4. The standard deviation is $8 \%$ demonstrating a good reproducibility when measuring a complex sample such as blood.
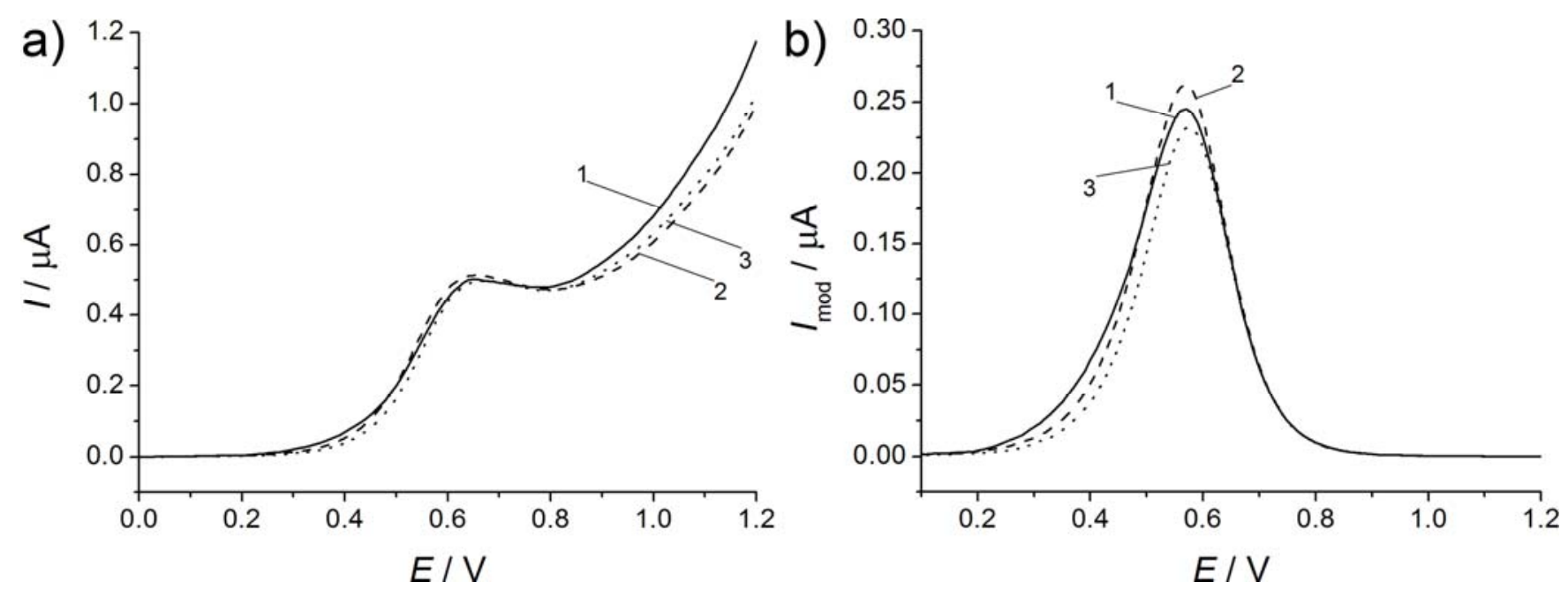

Fig. SI-4. Three LSVs of one blood sample from a female test person carried out in a triplicate measurement (a) and their corresponding PTVs (b). Start potential $0.0 \mathrm{~V}$ vs. Ag/AgCl, potential scan rate $100 \mathrm{mV} \mathrm{s}^{-1} . E_{\text {threshold }}=0.6 \mathrm{~V}, \lambda=0.5$.

Table SI-4. Antioxidant power of blood $\left(\mathrm{AOP}_{\text {blood }}\right)$ from a female test person. $\mathrm{AV}=$ Average, $\mathrm{SD}=$ Standard deviation.

\begin{tabular}{ccccc}
\hline Meas. 1 & Meas. 2 & Meas. 3 & AV & SD \\
\hline $56.80 \mathrm{nW}$ & $55.96 \mathrm{nW}$ & $48.52 \mathrm{nW}$ & $53.76 \mathrm{nW}$ & $4.5 \mathrm{nW}$ \\
\hline
\end{tabular}

